\title{
Content of foreign language (French) training at Agrarian university
}

\section{[Le composant professionnalisant du francais langue etrangere a l'Universite agraire]}

\author{
Aleksei Yu. Alipichev - Irina P. Gotovtseva - Olga V. Takanova
}

DOI: 10.18355/XL.2019.12.01XL.05

\begin{abstract}
The paper considers the career guiding role and pragmatic value of the "French as Foreign Language (FFL)" course at an agrarian university. In this context, the "French as Foreign Language" course can be aimed at not only the development of linguistic competence in the professional field but may also serve as a means of career guidance for students and the implementation of the career guiding principle of bachelor training. This course can be professionally advantageous in terms of smartly selected content that could motivate and facilitate the self-actualization of young graduates in their professional life. A new educational approach has been developed at Russian Timiryazev State Agrarian University to effectively achieve this integrating goal. This approach consists in designing the career guiding component of the FFL teaching content on the basis of the specific selected criteria. The implementation of this innovative component also requires specific processes that ensure the effectiveness of its motivating capacity for the professional activity. Career guidance is implemented through the selection of a relevant content component based on adequate requirements and effective ways of its enhancement. Owing to the offered requirements for the selection of career guiding component of university teaching content, the author has determined conditions for efficient organization and actualization of career guiding component of the "French as Foreign Language" course taught at agrarian universities, which will, consequently, help students to fit better into the world of work.
\end{abstract}

Key words: career awareness, career guiding component of teaching content, general curriculum subjects, motivation French as foreign language, agrarian university

\section{Résumé}

Cet article traite de la capacité professionnalisante en ce qui concerne une discipline générale comme "langue étrangère », et notamment pour le français à l'Université agraire. Dans ce contexte, le but du français langue étrangère (FLE) comprend non seulement la formation de la compétence communicative, mais également l'orientation professionnelle. Il est possible de bénéficier de ces avantages professionnalisants en sélectionnant intelligemment le contenu qui pourrait motiver et favoriser l'insertion des jeunes diplômés dans leur vie professionnelle.

Une nouvelle approche pédagogique a été élaborée à l'Université agraire d'Etat de la Russie Timiriazev, pour réaliser effectivement cet objectif. Cette approche consiste à concevoir un composant professionnalisant en matière de FLE, sur base de critères rationnels spécifiques. La mise en œuvre de ce composant innovant exige aussi des procédés particuliers qui assurent l'efficacité de son influence, motivante pour l'activité professionnelle. Grâce à l'introduction du composant professionnalisant en FLE, il est possible de donner plus de relief au sens et à la motivation du choix de telle ou telle filière par des étudiants et, in fine, de les aider à mieux s'insérer dans le monde du travail.

Mot clés : orientation professionnelle, composant professionnalisant, discipline générale, motivation, français langue étrangère, Université agraire 


\section{Introduction}

En raison de la modernisation de l'enseignement supérieur en Russie, l'apprentissage des langues dans les universités non linguistiques diminue tout en cédant la place aux disciplines liées au futur métier. En particulier, et actuellement à l'Université agraire d'Etat de la Russie Timiriazev - Académie agricole Timiriazev, des étudiants apprennent une langue étrangère pendant trois semestres au lieu de quatre. Dans ce contexte, il faut reconsidérer le contenu de la discipline générale " langue étrangère » - notamment le français - dont l'objectif principal est la formation de la compétence communicative. Néanmoins, il faut prendre en compte que la capacité en matière de langue étrangère n'est pas limitée par cet aspect.

Selon l'approche socio-psychologique, les critères de l'aptitude au travail sont: 1) les connaissances, les compétences et les acquis nécessaires ; 2) l'attitude envers la formation ; 3) la présence d'une pensée développée, indépendante et créative ; 4) la compréhension des tâches posées par la société ; 5) la conscience des motivations de ses activités, une compréhension claire de ses intérêts, inclinations, capacités ; 6) la corrélation des objectifs personnels et sociaux (Zimnyaya, 2019). Il est à constater que la volonté de l'individu de mener une vie active s'exprime à travers le système des qualités nécessaires à l'exercice des fonctions productives et sociales.

En d'autres termes, le contenu de la préparation des étudiants à leur activité professionnelle future est déterminé par le besoin des spécialistes dans la société qui sont capables de résoudre les problèmes professionnels actuels, en tenant compte du niveau moderne du développement de la production, en fixant de manière indépendante les objectifs du développement professionnel, en répondant au besoin de mise à jour ainsi que d'expansion continue des connaissances. En outre, ils doivent avoir un niveau élevé de développement en matière de pensée professionnelle, ainsi que des qualités psychophysiologiques, en raison de la nécessité de projeter sa volonté à long terme dans diverses conditions d'activité, etc. (Klimov, 1998).

L'enquête des employeurs a permis d'établir que dans les nouvelles conditions socioéconomiques, les exigences suivantes deviennent les plus importantes pour les diplômés universitaires :

1) une motivation formée pour l'activité professionnelle, ainsi que la conscience de soi socioprofessionnelle, et le désir du développement personnel et professionnel,

2) la connaissance de la spécialité et des domaines connexes, ainsi que le désir de maîtriser les nouvelles technologies professionnelles,

3) la capacité d'exercer des tâches dans les conditions de responsabilité personnelle, évaluation critique développée de soi et des autres,

4) les compétences communicatives formées (y compris la connaissance de langues étrangères - mise en œuvre de divers types de lecture pour comprendre des informations importantes sur le plan professionnel, connaissance et application des règles et normes de la correspondance commerciale avec des partenaires étrangers (expression écrite), présentation et compétences de base en communication professionnelle (expression orale), participation à une conversation ou une discussion (compréhension orale et expression orale).

De toute évidence, ces exigences amènent à l'élaboration d'approches novatrices de la formation linguistique dans le système d'enseignement professionnel. À notre avis, les éléments du contenu et les technologies, mises en œuvre dans le cadre du FLE (techniques, méthodes, formes, unités sémantiques, éléments opérationnels (opérations cognitives: analyse, synthèse, interprétation) et structures organisationnelles (hiérarchies de concepts, connexions logiques, etc.) ont une capacité de motivation. Ils contiennent les conditions préalables à la formation d'éléments de compétence et sont en même temps le moyen de projeter des éléments d'activité professionnelle à ceux d'activité universitaire. Dans le même temps, en 
raison de la nature complexe de l'activité professionnelle et des moyens de la motiver, l'effet le plus important est obtenu grâce à la synergie de tous les éléments de contenu et de technologie. Les exemples incluent une combinaison de projets et d'éléments de contenu nouveaux et significatifs sur le plan professionnel, des jeux d'entreprise, des présentations et des débats (présenter et défendre son opinion), ainsi que la recherche et la justification de moyens efficaces pour cela.

Alipichev et al. (2017) trouvent que le contenu devrait inclure une étude approfondie de la langue et une terminologie spéciale visant à élargir leur vision du monde interculturel, plus d'opportunités de carrière et de formation avancée, une motivation accrue dans les activités de formation et de recherche, l'étude plus consciente des disciplines, une expérience enrichie d'activités de formation quasi professionnelles et, bien sûr, une meilleure compréhension de ses compétences professionnelles et linguistiques. Kubrushko et al. (2018) proposent une approche de l'intégration des technologies intelligentes dans le contenu de la formation aux universités non linguistiques.

Le développement des compétences linguistiques par le travail avec des informations linguistiques, stratégiques, culturelles s'accompagne du développement des capacités cognitives, de la formation de compétences stratégiques, invariantes pour tout aspect de l'activité professionnelle. Les compétences professionnelles clés sont :

1) préliminaires: capacité de localisation socioprofessionnelle et de prise de conscience du niveau actuel de résolution de problèmes ; la capacité d'identifier les obstacles et les ressources, d'élaborer des stratégies et des tactiques de comportement, de prédire les résultats des activités, de diagnostiquer les écarts actuels, de prévenir les scénarios négatifs, de rechercher des solutions efficaces ;

2) informatives et discursives : développement d'informations provenant de différents domaines éducatifs (recherche, collecte, transfert, systématisation, traitement, stockage, distribution et autres opérations liées à des textes professionnels) ;

3) instrumentales et opérationnelles: possession de technologies et de moyens d'activités sociales et professionnelles ;

4) d'adaptation et de régulation: la capacité d'adaptation aux nouvelles conditions socioprofessionnelles, la gestion de ses activités et des activités d'autrui, la maîtrise du mécanisme d'adaptation aux niveaux comportemental, émotionnel et cognitif, et l'utilisation de l'expérience sociale et professionnelle pour résoudre de nouvelles tâches socioprofessionnelles dans certaines conditions ;

5) du contrôle, c'est-à-dire la comparaison du plan et du résultat, mise à l'échelle, etc. (Alipichev, 2014).

Selon Birova, J. Et al. (2017), pendant le processus d'apprentissage, les étudiants doivent développer leurs connaissances, leurs compétences, leurs attitudes multilingues et multiculturelles. Les changements mondiaux dans les domaines de l'information, de la communication, professionnels et autres, ont permis d'identifier de nouveaux domaines du système éducatif.

De plus, l'une des tâches principales est la formation des capacités des étudiants à extraire des informations utiles des textes professionnels étrangers, ainsi que d'organiser leurs activités dans le traitement des sources en langue étrangère, souvent en conditions de manque du temps (Alipichev et al, 2017).

\section{Revue de littérature}

De nombreux chercheurs ont développé les bases théoriques de la mise en œuvre du principe d'orientation professionnelle à l'université non linguistique. Ye.A. Klimov (Klimov, 1998) se concentre sur le lien entre l'orientation professionnelle et la formation du parcours professionnel de l'individu, ainsi que sur l'augmentation du niveau d'expression des qualités d'importance professionnelle des futurs spécialistes. B.A. Fedorishin (1987) identifie des éléments d'orientation professionnelle comme l'autodétermination et l'épanouissement professionnel. S.V. Yeregina (2006) justifie 
les fonctions d'orientation professionnelle et ses critères de manifestation chez des étudiants. E.N. Polianskaya (2008) explore les caractéristiques typiques de la persévérance, de l'initiative et des orientations de carrière des étudiants. Dans ses recherches, O.A. Rubtsova (2008) considère l'orientation professionnelle dans le système d'enseignement supérieur comme un outil facilitant la transition «étudetravail». B.A. Ospanova (1985) et R.G. Kiladzé (1989) examinent l'orientation professionnelle dans l'agriculture. O.V. Mirzabekova (2008) analyse l'application du principe d'orientation professionnelle dans le processus de formation du personnel d'ingénierie. O.G. Mokrinskaya (2006) explore l'humanisation de l'enseignement supérieur en tant que facteur de l'amélioration de sa qualité. N.A. Koroleva explore la structure du contenu et l'organisation de la formation humanitaire d'étudiants des universités techniques. Kulamikhina, Birova, Alipichev, Vasbieva, Kalugina (2018) examinent l'importance des compétences en matière de pensée critique pour le développement de l'orientation professionnelle des étudiants, en appliquant les stratégies incluses dans le programme de formation FLE. Certains chercheurs étudient le développement des compétences communicatives professionnelles des étudiants dans les établissements d'enseignement supérieur à l'aide de recherches thématiques, de présentations et de diverses activités interactives (Birova et al., 2016 ; Frolova et al, 2016 ; Kubrushko et al., 2013). Par conséquent, certains procédés pédagogiques devraient être révisés pour refléter les dernières réalisations en matière de théorie et de pratique de l'enseignement des langues étrangères dans les universités non linguistiques. A.K. Kroupchenko (2007), dans son étude, se concentre sur la méthodologie de professionnalisation de la formation linguistique. A.Yu. Alipichev (2014), A.N. Kuznetsov (2009), I.Z. Novoselova (1975) et d'autres ont mis au point la structure du contenu de la formation professionnelle en langues destinée aux étudiants des universités agraires. Cependant, sur base de l'analyse des travaux concernant le sujet de recherche, on peut affirmer que jusqu'à présent aucune solution n'a été trouvée à de nombreuses questions de la théorie et de la pratique de l'enseignement professionnel, notamment :

1) le potentiel d'orientation professionnelle des disciplines de l'enseignement général, notamment le FLE, n'est pas actualisé ;

2) il n'y a pas de critères pour évaluer l'orientation professionnelle des étudiants ;

3) les critères de la sélection du contenu professionnalisant des disciplines générales à l'université non linguistique, ne sont pas complètement développées.

\section{Matériaux et Méthodes}

Pour collecter des données empiriques, l'analyse théorique de la littérature philosophique, psychologique et pédagogique sur le sujet de recherche a été appliquée ; l'analyse du matériel pédagogique pour les universités agraires ; l'étude des documents réglementaires ; analyse de la théorie et de la pratique de l'éducation ; prévision scientifique ; observation ; enquêtes des étudiants. Pour le traitement des données empiriques, des méthodes de modélisation pédagogique, ainsi que le traitement de données statistiques et leur interprétation ont été utilisés ; le travail expérimental sur la validation du contenu professionnalisant du FLE à l'Université agraire a été réalisé.

L'étude a été menée en trois étapes.

Lors de la première étape, le choix de l'objet, du sujet et du problème de recherche, ainsi que l'analyse théorique ont été effectués ; la base de recherche et sa méthodologie ont été déterminées, l'hypothèse a été formulée ; les exigences modernes en matière de formation professionnelle à l'Université agraire et d'enseignement du FLE ont été analysées.

$\mathrm{Au}$ cours de la deuxième étape, l'hypothèse de recherche et les exigences supplémentaires relatives à la formation professionnelle d'étudiants des universités 
agraires ont été clarifiées et concrétisées; des outils de formation ont été mis au point; le matériel didactique innovant a été appliqué; l'expérience pédagogique a été lancée. À la troisième étape, l'analyse et la synthèse des résultats de l'expérience pédagogique ont été effectuées; l'analyse de l'efficacité de l'application de la méthodologie du FLE a été fait.

\section{Résultats et discussion}

Comme le montre l'analyse de la pratique actuelle de la formation en langues étrangères dans un certain nombre d'universités non linguistiques, le potentiel de compétence professionnelle du FLE n'a pas d'attention spécifique requise. En conséquence, le temps d'étude, déjà limité, est consacré à des activités parfois inadéquates pour la bonne mise en œuvre des fonctions professionnelles des diplômés (par exemple, la lecture à haute voix et la traduction ultérieure des textes éducatifs, la relecture, l'apprentissage des réalités professionnelles dans une langue étrangère).

Conformément à ce point de vue, une grande attention devrait être accordée à la variation du contenu objectif des tâches de formation (Smirnov, 2001). En raison de l'orientation thématique de l'apprentissage, les connaissances des réalités professionnelles, exprimées en langue étrangère, ainsi que les compétences professionnelles, sont combinées avec les aptitudes et compétences effectives en matière de communication professionnelle. Dans le même temps, les programmes manquent d'éléments issus de l'analyse didactique de la sphère de l'activité professionnelle d'éléments visant à les méthodes et techniques de mise en œuvre.

Ye. A. Klimov (1998), célèbre psychologue russe, a proposé des diverses étapes de la professionnalisation de la personnalité : «l'optant» désigne une personne en étape du choix du métier; "l'adepte» est une personne qui commence à maitriser son métier; l'adaptant est une personne expérimentée qui entre dans les détails de la profession ; «l'internale » est une personne qui est capable d'effectuer la plupart des tâches professionnelles de manière autonome.

En tenant compte de l'expérience pédagogique, on peut affirmer qu'au début des études universitaires, des étudiants sont encore optants, ils cherchent à comprendre et à analyser leur développement identitaire, ainsi que leur parcours professionnel. C'est en première et en deuxième année que des étudiants essaient de réaliser si leur choix de métier est valide ou non. La motivation au futur travail est influencée par un facteur comme la satisfaction des études universitaires, ce qui est à son tour étroitement lié au contenu des disciplines enseignées.

Dès lors, dans l'enseignement supérieur, l'orientation professionnelle est un principe prépondérant de la formation des spécialistes qui permet de varier son contenu, ses méthodes, ses formes et ses moyens, en fonction des conditions économiques et des exigences de la société, au niveau professionnel des employés.

En même temps, dans ces recherches scientifiques, il convient de distinguer deux concepts d'orientation professionnelle. L'orientation professionnelle est un processus visant à la formation de l'orientation professionnelle en tant que trait de personnalité. Dans l'interprétation de l'orientation professionnelle en tant que trait de personnalité, l'auteur utilise la définition proposée par S.V. Yeregina (2006): comportement actualisé et formé de l'ensemble des propriétés, des croyances de la personne, identifiées et améliorées par la formation professionnelle, ainsi que de l'ensemble de l'éducation et l'expérience, qui déterminent le besoin constant d'exercer des activités fondamentales et transversales professionnelles, et de développer les compétences. Cette définition devrait être élargie par l'inclusion d'éléments concernant la vision du monde professionnel, tels que des valeurs et des idées sur l'activité professionnelle.

Ainsi, si on limite l'objectif du FLE au sein de l'université non linguistique à la formation de compétences communicatives dans la sphère professionnelle, il y a un risque non seulement de déceler insuffisamment le potentiel de cette discipline 
générale, mais également de ne pas renforcer l'autodétermination professionnelle des étudiants (Krupchenko \& Kuznetsov, 2014).

Afin d'étudier l'état actuel de la mise en œuvre du principe d'orientation professionnelle à l'université lors de la préparation en langue étrangère, les manuels français recommandés pour le premier cycle par le programme de base ont été analysés. Il faut noter que le matériel pédagogique de la première étape de l'apprentissage du FLE nécessite des transformations significatives, c'est-à-dire qu'il doit viser initialement la formation d'une orientation professionnelle.

Compte tenu de la situation de réduction des heures d'apprentissage des langues étrangères à l'Université agraire, il est nécessaire dès les premières cours d'orienter professionnellement les étudiants, en influençant la sphère de leurs intérêts, de leurs intentions et de leurs valeurs. Cet objectif implicite est atteint grâce à une sélection minutieuse du contenu, reflétant les principaux concepts de l'orientation professionnelle, c'est-à-dire la «signification sociale $\mathrm{du}$ travail» et les «caractéristiques de la future activité professionnelle». $\mathrm{Ce}$ composant professionnalisant du contenu peut être inclus au processus général d'enseignement d'une langue étrangère, créant ainsi des conditions favorables à la formation des qualités professionnelles du futur spécialiste, notamment son aptitude psychologique et sa motivation à exercer des activités professionnelles.

Les critères les plus appropriés et les plus avérés de la sélection du composant professionnalisant du FLE, dont l'efficacité est prouvée à l'Université agraire, sont les suivants:

- l'accessibilité cognitive, qui signifie la capacité des étudiants à assimiler le composant professionnalisant du contenu en tenant comte de la zone proximale du développement ;

- l'efficacité et rationalité du contenu pour l'autodétermination professionnelle, le développement des qualités recherchées, de la mentalité professionnelle, l'encouragement de la maîtrise de la future profession et l'adaptation aux particularités de l'activité professionnelle en tenant comte des motifs actuels du choix du métier, des valeurs des étudiants, et en supprimant tout ce qui est secondaire du contenu professionnalisant ;

- la validité de la matière communicative et du discours professionnel, qui représente leur signifiance par rapport aux sphères, sujets, situations de la communication professionnelle couvrant éventuellement tout le champ cognitif de la formation de l'orientation professionnelle (Takanova, 2009).

Après la sélection du composant professionnalisant, un aspect important est son actualisation efficace, ce qui implique le choix des procédés opportuns de présenter des informations aux étudiants. Dans ces recherches trois méthodes principales sont proposées pour garantir l'impact pédagogique longitudinal lors de l'organisation de l'orientation professionnelle par l'intermédiaire $\mathrm{du}$ contenu composant professionnalisant :

- la permanence - le flux continu d'informations professionnalisantes reflétées dans les sphères conceptuelles «signification sociale du travail» et «caractéristiques de la future activité professionnelle»;

- la cohérence (de lat. cohaerens - être en communication) - cohérence et harmonie de la présentation de la matière communicative et du discours professionnel sélectionnée, ce qui implique un impact informationnel temporel sur chaque élève du groupe;

- la linéarité - séquence de présentation bien structurée de la matière communicative et du discours professionnel, assurant une continuité de communication ainsi qu'une orientation professionnelle stable et prévisible de la structure et du concept du composant professionnalisant du contenu proposé, afin d'éviter toute distorsion de sa dominante sémantique.

XLinguae, Volume 12 Issue 1XL, January 2019, ISSN 1337-8384, eISSN 2453-711X 
Le respect des critères ci-dessus pour la sélection du composant professionnalisant et des procédés de son actualisation garantit l'immanence de l'orientation professionnelle, c'est-à-dire la fusion, le lien interne de la conscience, la sphère émotionnelle et sensuelle de la personnalité avec la future profession. À son tour, l'orientation professionnelle est un paramètre permettant d'évaluer le contenu professionnalisant à l'université.

A la base de ces critères a été développé le contenu du manuel pratique typique à orientation professionnelle pour FLE, dont l'application expérimentale a été réalisée de 2015 à 2018. Plusieurs universités non linguistiques en Russie ont confirmé l'efficacité et la faisabilité des critères qui ont été proposés pour évaluer les critères de la sélection du composant professionnalisant. Lors de la conception de ce manuel pratique, une attention particulière a été accordée à la mise en œuvre des principes de faisabilité pratique et de motivation globale. L'approche par compétences a été utilisée comme base méthodologique.

Le manuel proposé se compose de deux parties. La première partie vise à la formation d'une attitude positive à l'égard du travail, et pose le problème du sens du travail. La seconde partie vise directement à développer une compréhension de la signification sociale, du prestige du métier d'ingénieur agricole, et à souligner son rôle dans le développement de l'agriculture. Dans cette partie, les motifs du choix de cette profession sont clarifiés, les avantages d'étudier à l'Université agraire sont soulignés, l'attention est centrée sur la polyvalence et les tâches des spécialistes de ce profil, sur les exigences professionnelles spécifiques, les qualités actuelles nécessaires à un jeune spécialiste en agro-ingénierie sont analysées, l'accent est mis sur les perspectives de travail dans l'agriculture et sa signification sociale.

Lors des études expérimentales, les étudiants répondent à la question: Comment pourrait être le travail? Pour y répondre, ils emploient le vocabulaire proposé pour caractériser le travail: nouveau, intéressant, important, bien payé et responsable. Cela veut dire que les étudiants se voient proposer un vocabulaire qui caractérise le travail exclusivement du point de vue positif. En outre, les étudiants, à la base de l'exercice précédent caractérisent le travail de l'ingénieur agricole. Ensuite les étudiants répondent à la question: Pourquoi les gens travaillent-ils? Ils réfléchissent aux raisons qui motivent les gens pour travailler. Les réponses proposées aux élèves sont suivantes: Les gens travaillent pour acheter quelque chose; faire une carrière; apprendre quelque chose de nouveau; établir des contacts; trouver la reconnaissance; pour en bénéficier, etc. Ensuite, les étudiants répartissent ces raisons en quatre groupes principaux: 1) gagner de l'argent; 2) profiter du travail; 3) aider les autres; 4) trouver la reconnaissance. Ces groupes sont liés aux besoins humains fondamentaux, à savoir, les besoins de reconnaissance, d'expression personnelle, et les besoins matériels et sociaux qui ont été identifiés dans ces recherches.

Après, les étudiants répondent à la question: Pourquoi voudriez-vous travailler? Pour répondre, ils peuvent employer des expressions telles que: Je dois contacter des personnes; Je me sens bien seulement quand je travaille; Bien sûr, à cause de l'argent; je suis heureux en profitant à quelqu'un, etc. Les étudiants sont amenés au problème de la signification du travail, car l'éducation devrait fournir un moyen de détecter les significations.

Dans le type d'exercice suivant, il est proposé aux étudiants de raconter avec quoi et avec qui ils ont déjà travaillé. Il y a des réponses telles que: personnes, outils, véhicules, équipement de bureau, animaux et plantes, électrotechnique, projets, langues étrangères, informations, appareils de mesure, etc. Il convient de noter que les étudiants discutent avec enthousiasme de leurs compétences, se comparent avec leurs camarades de classe et essaient d'être les meilleurs, mais partagent également une expérience de travail qui peut ensuite leur être utile dans le domaine de l'agroingénierie. 
Après, les étudiants répondent aux questions suivantes: Qu'est-ce qui est important pour vous dans votre travail? Quel est le travail parfait pour vous? Avant d'exprimer leurs points de vue, les étudiants prennent connaissance avec les opinions de trois spécialistes. Dans cette tâche, le polylogue est utilisé consciemment. Les étudiants se sentent donc immergés dans la situation et ils expriment leurs propres opinions, en utilisant des mots de référence tels que: tout d'abord, je souhaite un salaire élevé, un lieu de travail stable, un programme social, un horaire de travail pratique, la carrière et le prestige sont importants pour moi, je souhaite travailler en équipe, avec des collègues étrangers, etc.; je ne veux pas travailler la nuit, avoir un petit salaire, un horaire de travail gênant, etc.

Dans le type d'exercice suivant, les étudiants continuent à travailler avec un polylogue, à savoir qu'ils prennent connaissance avec le point de vue de quatre diplômés de l'Université agraire concernant leur formation. Afin de mieux intérioriser les informations proposées, les étudiants, lors de la lecture et de la traduction des énoncés, remplissent un tableau comprenant les colonnes suivantes: 1) le nom du diplômé; 2) le domaine professionnel; 3) les avantages de la formation en ingénierie agroalimentaire. Les diplômés de l'Université agraire ont jusqu'à 30 ans et travaillent avec succès dans le domaine de la vente de machines agricoles, de l'énergie et de l'industrie automobile, participent à des travaux de conception et de recherche dans le domaine de l'agriculture. Dans leurs déclarations, les diplômés accordent une attention particulière à la polyvalence des spécialistes en ingénierie agroalimentaire, à la possibilité d'ouvrir leur propre entreprise dans le domaine de l'agriculture, à la promotion de carrière, à la coopération avec des collègues étrangers, etc. En même temps, tout est possible grâce à la formation à l'Université agraire, qui fournit toutes les connaissances et les compétences nécessaires.

La deuxième partie du manuel expérimental vise directement à orienter les étudiants vers des activités d' agro-ingénierie.

Les étudiants répondent à la question: Pourquoi voulez-vous devenir ingénieur agricole? Pour répondre, ils utilisent des phrases de référence qui reflètent les principales motivations du choix de cette profession, telles que: Je voudrais devenir ingénieur parce que 1) j'en ai toujours rêvé; 2) mes parents aiment cette profession; 3) je voudrais travailler avec des appareils; 4) les ingénieurs sont toujours en demande; 5) il y a de bonnes perspectives professionnelles; 6) ce métier est très important, intéressant; 7) je peux appliquer mes compétences professionnelles partout, etc. Il convient de noter que cette étape de l'application pratique montre déjà que les étudiants choisissent le plus souvent des phrases telles que : j'aimerais travailler avec la technologie; les ingénieurs sont toujours en demande; il y a de bonnes perspectives professionnelles. Ceci indique indirectement la dynamique positive du classement individuel des qualités personnelles significatives sur le plan professionnel.

L'exercice suivant présente un dialogue entre un étudiant de l'Université agraire et ses connaissances - un candidat potentiel de l'Université agraire. Les étudiants, tout en lisant et en traduisant ce dialogue, réfléchissent à une réponse à la question suivante: quelles sont les possibilités d'épanouissement professionnel offertes par une formation à l'Université agraire? Le dialogue porte sur les possibilités suivantes: 1) l'étude des disciplines réellement nécessaires aux futures activités professionnelles, y compris une langue étrangère; 2) stage à l'étranger (qui donne une expérience dans le domaine de l'ingénierie agroalimentaire); 3) l'établissement de contacts professionnels et personnels lors de la visite d'expositions agricoles spécialisées. De ce fait, l'étudiant comprend qu'il a correctement choisi la profession et l'université ; après cela, il deviendra un spécialiste qualifié et recherché, et pourra trouver un emploi stable et bien rémunéré.

XLinguae, Volume 12 Issue 1XL, January 2019, ISSN 1337-8384, eISSN 2453-711X 
Après, les étudiants lisent le texte «Ingénierie agroalimentaire a de bonnes perspectives pour les spécialistes», qui traite de l'universalité des spécialistes dans le domaine de l'agro-ingénierie, de leurs tâches principales et des exigences de ce métier. Tout en lisant et en traduisant un texte, les élèves remplissent un tableau qui met en valeur les tâches d'un ingénieur agricole en tant que gestionnaire, consultant, ses tâches dans l'industrie et la recherche.

A la base du contenu du texte, les étudiants répondent aux questions suivantes: 1) Le métier d'ingénieur agricole correspond-il à vos idées? 2) Quelles sont les exigences de cette profession? 3) Quelles sont vos connaissances et votre expérience dans le domaine de l'ingénierie agricole? 4) Quelles sont les tâches de l'ingénieur agricole qui vous intéressent le plus?

Ensuite les étudiants analysent un diagramme «La demande des agro-ingénieurs est extrêmement forte», reflétant la demande croissante de données de spécialistes en marketing, recherche et conception, production agricole, services et conseil. Ils répondent aux questions: Quelle conclusion pouvez-vous tirer de ce diagramme? Quelles sont vos chances de trouver un emploi après vos études à l'université d'agroingénierie? Pour répondre aux étudiants, des phrases ayant un contenu positif ont été délibérément offertes: j'estime qu'après mes études à l'université, j'ai d'excellentes chances de trouver un emploi ; je vais facilement (rapidement, cent pour cent) trouver un emploi.

Dans le type d'exercice suivant, les étudiants travaillent avec un dialogue entre un spécialiste d'un profil en agro-ingénierie, et un étudiant de l'Université agraire. Le dialogue discute des qualités importantes sur le plan professionnel qui sont nécessaires pour un jeune spécialiste qui souhaite trouver du travail avec succès dans le domaine de l'agro-ingénierie. Ces qualités incluent la capacité de travailler en équipe, le talent organisationnel, la pensée analytique, les compétences en informatique, les compétences en langues étrangères, la créativité, la compétence, la motivation, etc.

Dans le type d'exercice suivant, les étudiants se voient proposer le texte : «La signification sociale de l'agriculture». Lors de la lecture et de la traduction d'un texte, les élèves doivent compléter un tableau analysant la signification sociale de l'agriculture en tant que producteur, environnementaliste, employeur et consommateur. En outre, sur la base du contenu de ce texte, les étudiants classent leurs activités en agriculture, par exemple: produire des aliments de haute qualité; produire du biogaz à partir de biomasse, du biodiesel à partir d'huile de colza; cultiver des plantes (élever des animaux); protéger l'environnement; travailler avec des machines agricoles; travail sur le terrain (dans le bâtiment d'élevage, dans le laboratoire); travailler avec un ordinateur, etc. Après cela, les élèves commentent leurs propres résultats.

Pour que les étudiants soient encore plus convaincus des perspectives d'emploi dans l'agriculture et qu'ils désirent devenir ingénieurs agricoles, un entretien avec un haut responsable du ministère de l'Agriculture de la Fédération de Russie est proposé. Au cours de la lecture et de la traduction du dialogue, les élèves cherchent des réponses aux questions suivantes: 1) Quelles activités le ministère de l'Agriculture de la Fédération de Russie considère-t-il comme des priorités? 2) Pour quels événements le ministère de l'agriculture de la Fédération de Russie attribue-t-il les allocations les plus importantes? 3) Quel type d'équipement agricole doit-il être produit en Russie? Les étudiants découvriront de ce dialogue le potentiel agricole de la Russie, le soutien par l'État du secteur agricole, la modernisation de l'agriculture, l'amélioration de l'éducation et de la formation avancée des travailleurs du secteur agraire, la réglementation des importations et des exportations; la solution des problèmes sociaux dans les zones rurales.

Dans l'exercice final, les étudiants remplissent un questionnaire de stage à l'étranger. Le questionnaire comprend les questions suivantes: 1) Quel est votre nom? 2) D'où 
venez-vous? 3) Où et qu'étudiez-vous? 3) Pourquoi voulez-vous devenir un ingénieur agricole? 4) Qu'est-ce qui vous intéresse particulièrement dans l'agriculture? 5) Quelles sont vos connaissances et votre expérience dans le domaine de l'ingénierie agricole? 6) Quel est votre niveau de maîtrise d'une langue étrangère, en particulier de la langue professionnelle? 7) Quelles sont vos qualités professionnelles importantes pour un stage réussi? 8) Que signifie le stage à l'étranger pour vous? Il est recommandé aux étudiants d'utiliser le maximum de vocabulaire appris et pour cela, il est fait référence aux exercices dans lesquels les étudiants peuvent choisir la réponse au questionnaire. Ainsi, les étudiants répètent une fois de plus le matériel étudié et intériorisent les informations d'orientation professionnelle, grâce auxquelles l'orientation professionnelle est formée.

Il convient de souligner que le composant professionnalisant du contenu joue un rôle de liaison entre les composants de formation de la personnalité et celui du discours professionnel, car il englobe deux concepts: l'importance sociale du travail et les caractéristiques de la future activité professionnelle. La subdivision de l'enseignement et de son contenu en général et spécial, avec l'enseignement polytechnique comme une branche spéciale de leur intersection. À son tour, cela implique une division de l'expérience sociale et personnelle dans la réalisation de différents types d'activités en fonction du degré de leur communauté: les activités réalisées par tous et les types d'activités spéciales. De même, le composant professionnalisant comprend deux parties: la formation de la personnalité (signification sociale du travail) et la professionnalisation (les caractéristiques de la future activité professionnelle), contribuant ainsi à la professionnalisation de l'individu.

Avant le développement de ce composant de liaison innovant, les composants du contenu des disciplines de la formation générale de l'Université agraire qui formaient la personnalité et étaient autonomes, ne permettaient pas aux étudiants de développer une orientation professionnelle et ne respectaient pas le principe d'orientation professionnelle. De plus, le FLE était apical, c'est-à-dire autonome dans la structure du contenu de la formation des spécialistes. Avec l'introduction du composant susmentionné, la langue étrangère est devenue une partie implicite de la formation, car elle fait partie de la formation professionnelle et devient la discipline de base pour la formation des compétences techniques générales des futurs ingénieurs agricoles.

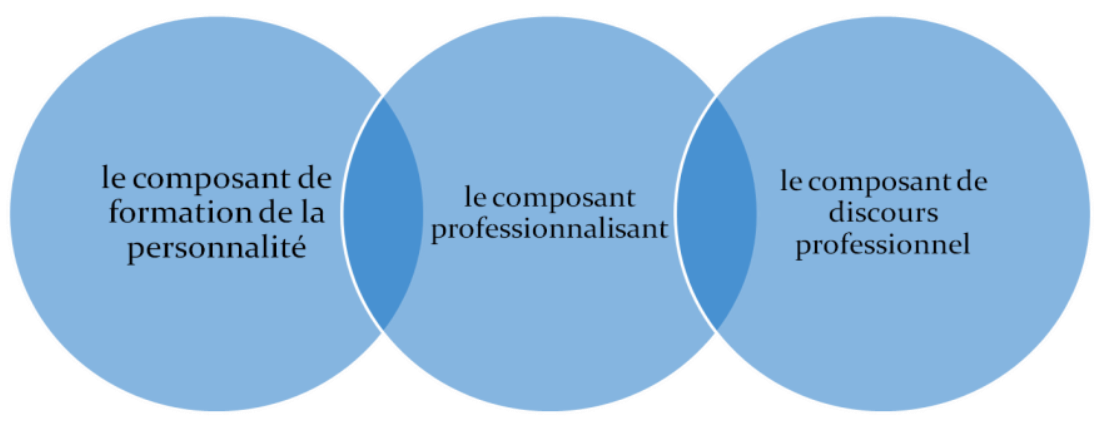

Figure 1 : Interaction des composants

Une formation expérimentale à la discipline «langue étrangère», basée sur l'utilisation des matériaux de ce manuel typique, a été lancée en 2015 avec des étudiants de 1ère et 2e années de l'Académie agricole K.A. Timiryazev. L'étude a porté sur 173 étudiants; 
l'échantillon limité est dû au petit nombre d'étudiants apprenant le français. Deux flux ont été formés: les groupes de contrôle comprenaient 53 personnes, les groupes expérimentaux 120 personnes.

Dans ces recherches, nous avons examiné la dynamique de l'évaluation individuelle des besoins de base, des valeurs et des motivations du choix de l'activité d'ingénierie agroalimentaire, qui ont été déterminés à l'aide de trois tests psychodiagnostiques standards.

Ainsi, le degré de satisfaction de cinq besoins fondamentaux des étudiants a été révélé: 1) besoins sociaux (interpersonnels); 2) besoins de sécurité; 3) besoins matériels; 4) besoins de reconnaissance; 5) le besoin d'expression de soi. Les tests ont montré qu'après avoir étudié le composant professionnalisant, le besoin de reconnaissance avait considérablement augmenté.

Tableau 1 : La dynamique de l'évaluation des besoins de base

\begin{tabular}{|l|c|c|c|}
\hline \multicolumn{1}{|c|}{ besoins } & $\begin{array}{c}\text { avant } \\
\text { expérimentation } \\
\text { (personnes \%) }\end{array}$ & $\begin{array}{c}\text { après expérimentation } \\
\text { groupe expérimental } \\
\text { (personnes \%) }\end{array}$ & $\begin{array}{c}\text { après } \\
\text { expérimentation } \\
\text { groupe de contrôle } \\
\text { (personnes \%) }\end{array}$ \\
\hline sociaux & 41,6 & 43,3 & 41,5 \\
\hline matériels & 35,8 & 29,2 & 37,8 \\
\hline de reconnaissance & 39,3 & 52,5 & 32,1 \\
\hline $\begin{array}{l}\text { d'expression de } \\
\text { soi }\end{array}$ & 50,3 & 41,7 & 56,6 \\
\hline de sécurité & 37,6 & 24,2 & 43,4 \\
\hline
\end{tabular}

Les étudiants sont plus motivés pour accroître leurs compétences afin de réussir à se réaliser dans le domaine de l'agroingénierie. Dans le même temps, les étudiants souhaitent toujours établir des contacts interpersonnels solides, ce qui contribuera également à leur épanouissement en tant qu'ingénieurs de l'agroalimentaire. Un résultat positif est également une diminution des besoins matériels et des besoins de sécurité des étudiants. Cela indique une augmentation de leur confiance en leur avenir et une diminution du niveau d'inquiétude face à un confort matériel, ce qui est la conséquence de la réception d'informations positives sur les perspectives de développement du complexe agro-industriel au cours d'une formation expérimentale.

La dynamique de l'évaluation individuelle des valeurs terminales et instrumentales des étudiants a été éxaminée à l'aide de la méthodologie psychodiagnostique de M. Rokitch (Raygorodskiy, 2008). Les résultats du test ont démontré une dynamique positive des valeurs directement liées à la professionnalisation de l'individu. Après avoir étudié le composant professionnalisant du contenu de la discipline générale «langue étrangère», l'un des objectifs prioritaires de la vie des sujets devient le désir d'exercer un travail intéressant. Parallèlement, les étudiants commencent à apprécier de telles démarches en toute situation, en matière d'efficacité et de productivité (Takanova, 2009).

Tableau 2 : La dynamique de l'évaluation des valeurs 


\begin{tabular}{|l|c|c|c|}
\hline Valeurs & $\begin{array}{c}\text { avant } \\
\text { expérimentation } \\
\text { place dans le } \\
\text { classement }\end{array}$ & $\begin{array}{c}\text { après } \\
\text { expérimentation } \\
\text { dans le groupe } \\
\text { expérimental } \\
\text { place dans le } \\
\text { classement }\end{array}$ & $\begin{array}{c}\text { après } \\
\text { expérimentation } \\
\text { dans le groupe } \\
\text { de contrôle } \\
\text { place dans le } \\
\text { classement }\end{array}$ \\
\hline terminales : & & & \\
\hline métier intéressant & 10 & 6 & 11 \\
\hline reconnaissance & 12 & 9 & 14 \\
\hline apprentissage & 13 & 12 & 13 \\
\hline productivité & 11 & 10 & 12 \\
\hline développement & 9 & 7 & 13 \\
\hline instrumentales: & & & 14 \\
\hline praticabilité & 11 & 9 & 15 \\
\hline rationalisme & 10 & 12 & \\
\hline efficacité & 12 & 9 & \\
\hline
\end{tabular}

La dynamique positive des motivations intérieurement significatives, significatives socialement, et des motivations positives externes atteste que, après avoir reçu les informations nécessaires et manquantes sur la future activité professionnelle, les étudiants sont convaincus que cela était intéressant pour eux et correspond à leurs capacités, donne des possibilités de faire preuve de créativité. En outre, cela prouve que les étudiants ont commencé à réfléchir davantage à la signification sociale et à l'universalité de la profession choisie. Ils sont convaincus que la profession choisie constitue une opportunité de faire carrière, et permet de développer des compétences en leadership. De plus, à la suite de l'étude du manuel innovant, les motifs du choix d'un métier parmi les étudiants sont devenus plus clairs, ce qui prouve la réduction de l'influence de plusieurs types de motifs sur le choix du futur métier en même temps. Il convient de noter comme résultat positif après l'expérience qu'il n'y avait aucune influence sur le choix du domaine d'activités des motifs négatifs externes, à savoir: des parents aiment cette profession, elle est choisie par mes amis, elle est la seule possible dans les circonstances.

Après avoir mené une formation expérimentale en langue étrangère, un questionnaire écrit anonyme destiné aux étudiants du groupe expérimental a été réalisé afin d'obtenir leur évaluation de ce travail pratique. Selon I.N. Shpilrein (1929), les données obtenues au cours de l'enquête par questionnaire peuvent être considérées comme assez objectives, car ce que la personne veut paraître nous donne certaines indications sur sa véritable identité.

A la première question du questionnaire : Qu'avez-vous appris de votre futur métier?, les réponses ont été les suivantes: ... le métier est très bien payé; J'ai appris que le métier est très prestigieux, il a un grand avenir!; J'ai appris que mon métier est important, plus important que je ne le pensais; ... Qu'il y a une grande demande pour cela! Et les diplômés de cet université sont très demandés ; j'ai appris que le métier d'ingénieur est fascinant, et que je vais travailler avec différentes techniques; ... qu' il sera possible d'effectuer un stage à l' étranger.

À la question : Votre désir de devenir ingénieur agricole s'est-il intensifié? Il y a eu des réponses positives telles que: Oui, je veux continuer à apprendre mon métier; Oui, depuis que j'ai été convaincu une fois de plus que ce métier est en demande.

Le travail expérimental réalisé confirme que le composant développée du contenu correspond à son objectif principal - la formation de l'orientation professionnelle, car XLinguae, Volume 12 Issue 1XL, January 2019, ISSN 1337-8384, eISSN 2453-711X 
elle contient l'information la plus complète et, surtout, celle qui manque aux étudiants du métier choisi. Les recherches relatives à l'efficacité de ces matériaux devraient se poursuivre.

\section{Conclusion}

En conclusion, il faut noter que l'analyse des sources scientifiques, des études théoriques et des travaux pratiques effectués ont confirmé l'hypothèse avancée. Conformément au but et aux objectifs de l'étude, ses principaux résultats théoriques et pratiques ont été formulés, les domaines prometteurs pour des travaux ultérieurs ont été identifiés, et les conclusions générales suivantes ont été formulées.

Le développement de l'orientation professionnelle des étudiants est un facteur essentiel pour assurer la qualité de la formation. L'orientation professionnelle initiale des étudiants peut s'effectuer au niveau du premier cycle dans le cadre de la préparation en disciplines générales, particulièrement en FLE. Dans le même temps, l'analyse a montré qu'actuellement, le potentiel professionnalisant de cette discipline n'est pas suffisamment actualisé.

L'orientation professionnelle préparant aux disciplines de l'enseignement général est une étape propédeutique de la formation en fonction de l'orientation professionnelle en tant que trait de personnalité, ainsi que de l'activité cognitive dans les disciplines professionnelles générales et spéciales; elle nécessite le développement du composant professionnalisant du contenu (Takanova, 2009).

En même temps, ces recherches ne sont pas exhaustives. Plus concrètement, les mécanismes psychologiques de la formation de l'orientation professionnelle par l'intermédiaire des disciplines générales ne sont pas complètement étudiés, et la question d'une application plus large du concept développé reste ouverte.

\section{Bibliographic references}

ALIPICHEV, A.YU. 2014. Optimizatsiya soderzhaniya i tekhnologii modul' nogo obucheniya inostrannym yazykam $\mathrm{v}$ podgotovke magistrov // Vestnik federal' nogo gosudarstvennogo obrazovatel' nogo uchrezhdeniya vysshego professional' nogo obrazovaniya"Moskovskiy gosudarstvennyy agroinzhenernyy universitet im. V.P. Goryachkin", No. 4. - pp. 103-106.

ALIPICHEV A.YU. - KHALEVINA S.N. - TRUBCHENINOVA A.A. - FEDULOVA A.N.

Practical solutions to foreign language training courses implemented using distance learning

IEJME: Mathematics Education. 2017. Vol. 12. n. 1, pp. 59-68. ISSN: 2468-4945

BIROVA, J. - KLIMOVA, I.I. - KALUGINA, O.A. 2016. Some critics on Language Education Assessment. In: IEJME: Mathematics Education. vol. 11, n. 7. Pp. 24702482. ISSN 2468-4945.

BIROVA, J. - VASBIEVA, D.G. - MASALIMOVA A.R. 2017. Communication in French foreign language learning by implementing the aspects of interculturality. In: Komunikacie, vol.19, n.4, pp. 95-104. ISSN: 1335-4205.

FEDORISHIN, B.A. 1987. Teoreticheskiye aspekty sovershenstvovaniya sistemy pedagogicheskoy oriyentatsii [Theoretical aspects of improving the system of pedagogical career guidance]. In: Oriyentatsiya molodezhi na pedagogicheskuyu professiyu. Poltava. $60 \mathrm{p}$.

FROLOVA, V.B. - KALUGINA, O.A. - ARTAMONOVA, L.S. - BOYKOV, A.I. 2016. Studying the Practice of Performance Assessment and Rating System Implementation in Higher Institutions. In: IEJME: Mathematics Education. vol. 11, n.5, pp. 1185-1193. ISSN 2468-4945

KILADZE, R.G. 1989 Pedagogicheskiye osnovy oriyentatsii shkol' nikov na sel' skokhozyaystvennyye professii [Pedagogical foundations of the career guidance of agricultual students]: Self-review of DSc (Ped) thesis. Tbilisi. $39 \mathrm{p}$. 
KLIMOV, YE.A. 1998. Vvedeniye $\mathrm{v}$ psikhologiyu truda [Introduction to the psychology of labor]. // Uchebnik dlya vuzov. - M.: Kul' tura i sport: Izd. ob-niye "Yuniti". 349 p.

KRUPCHENKO, A.K. 2007. Stanovleniye professional' noy lingvodidaktiki kak teoretiko-metodologicheskaya problema $\mathrm{v}$ professional' nom obrazovanii [Formation of professional linguodidactics as a theoretical and methodological problem in professional education]: DSc (Ed) thesis. Moscow 555 p.

KRUPCHENKO, A.K. - KUZNETSOV, A.N. 2014. Sovremennyy uroven' i perspektivy razvitiya professional' noy lingvodidaktiki [Current state and prospects for the development of professional linguodidactics]. In: Vestnik Federal' nogo gosudarstvennogo obrazovatel' nogo uchrezhdeniya vysshego professional' nogo obrazovaniya "Moskovskiy gosudarstvennyy agroinzhenernyy universitet im. V.P. Goryachkina. No. 1. pp. 76-81.

KUBRUSHKO P.F. - NAZAROVA L.I. Professional Development of Technical University Lecturers in Field of Innovation Teaching. The Global Challenges in Engineering Education: Proceedings of the 42 International IGIP Symposium, 25-27 September 2013. Kazan: Kazan National Research Technological University, 2013, pp. 467-469. 978-1-4799-0152-4/13/\$31.00 @ 2013 IEEE

KUBRUSHKO P. - SHISHOV S. - KALNEI V. - SCARAMANGA V. SHAFAZHINSKAYA N. - RABADANOVA R. Perception of educational information in the process of learning of construction and humanitarian universities students: comparative analysis. International Journal of Civil Engineering and Technology (IJCIET), Volume 9, Issue 11, November 2018, pp. 2331-2337, Article ID: IJCIET_09_11_232. Available online http://www.iaeme.com/ijciet/issues.asp?JType=IJCIET\&VType=9\&IType=11 ISSN Print: 0976-6308 and ISSN Online: 0976-6316

KULAMIKHINA, I.V. - BIROVA, J. - ALIPICHEV, A.YU. - VASBIEVA, D.G. KALUGINA O.A. 2018. Developing Communication and Critical Thinking through Creative Writing in English and French Language: Analysis of Classroom Management Strategies. In: Komunikacie. vol. 20, n.1, pp. 115-130. ISSN 1335-4205. MIRZABEKOVA, O.V. 2008. Professional' naya napravlennost' obucheniya spetsialistov na osnove resheniya tipovykh zadach [Career guidance in specialists' training on the basis of typical problem solving]. - Chelovek i obrazovaniye, n. 2 (15). pp. 40-43.

MOKRINSKAYA, O.G. Gumanitarizatsiya meditsinskogo obrazovaniya kak faktor povysheniya kachestva obucheniya $\mathrm{v}$ vuze: Na primere izucheniya inostrannogo yazyka [Humanitarization of medical education as a factor in improving the quality of higher education: A case of foreign language learning]: $\mathrm{PhD}(\mathrm{Ed})$ thesis. Volgograd, 2006. 243 p.

NOVOSELOVA, I.Z. 1975. Ispol' zovaniye TSO pri izuchenii IYA v neyazykovykh vuzakh [Using techical aids in foreign language teaching in non-linguistic universities]. - M.: NII problem vysshego obrazovaniya. $36 \mathrm{p}$.

OSPANOVA, B.A. 1985. Professional' naya oriyentatsiya shkol' nikov na sel' skokhozyaystvennyy trud $\mathrm{v}$ uchebnom protsesse. (Na materiale izucheniya distsiplin yestestvennogo tsikla v 5-10 kl.) [Career guidance of schoolchildren to farming activities during the study process. (Based on the study of natural cycle courses in 510 forms)]: PhD (Ed) thesis. Alma-Ata. 219 p.

POLYANSKAYA, YE.A. 2008. Individual' no-tipicheskiye osobennosti nastoychivosti, initsiativnosti i kar' yernykh oriyentatsiy studentov [Individuallytypical features of perseverance, initiative and career preferences of students]: Selfreview of PhD (Psy) thesis. M. $22 \mathrm{p}$.

RAYGORODSKIY, D.YA. 2008. Prakticheskaya psikhodiagnostika [Practical psychodiagnostics]. - Izdatel' skiy dom "BAKHRAKH", Samara, 670 p. 
RUBTSOVA, O.A. 2008. Proforiyentatsiya v sisteme vysshego obrazovaniya kak instrument sodeystviya perekhoda "ucheba - rabota" [Career guidance in the higher education system as a tool for facilitating the "study-work" transition]: $\mathrm{PhD}$ (Econ) thesis. - M. $191 \mathrm{p}$.

SHPILREYN, I.N. Rukovodstvo po psikhotekhnicheskomu professional' nomu podboru [Guidelines for psychological-and-technical professional selection]. - Gos. izd-vo, 1929. pp. 346-348.

SMIRNOV, S. D. 2001. Pedagogika i psikhologiya vysshego obrazovaniya: ot deyatel' nosti $\mathrm{k}$ lichnosti [Pedagogy and psychology of higher education: from activity to personality]. - M. : ITS "Akademiya". $304 \mathrm{p}$.

TAKANOVA, O.V. 2009. Razrabotka professional' no oriyentiruyushchego soderzhaniya obshcheobrazovatel' nykh distsiplin $\mathrm{v}$ agroinzhenernom vuze [Development of career guiding content of general education subjects at an agricultural engineering university]: $\mathrm{PhD}(\mathrm{Ed})$ thesis. $210 \mathrm{p}$.

TAKANOVA, O. V. 2016. Trebovaniya $\mathrm{k}$ otboru professional' no oriyentiruyushchego komponenta soderzhaniya distsipliny inostrannyy yazyk [Requirements for the selection of career guiding component of the teaching content of the "Foreign Language" course]. In: Sbornik materialov ochno-zaochnoy regional' noy nauchno-prakticheskoy konferentsii s mezhdunarodnym uchastiyem. - Omsk, Izd. FGBOU VO Omskiy GAU. pp. 94-96

TAKANOVA, O. V. - KUZNETSOV, A. N. 2009. Psikhologo-pedagogicheskiye faktory professional' noy oriyentatsii studentov agrarnykh vuzov sredstvami distsipliny "Inostrannyy yazyk" [Psychological and pedagogical factors of career guidance of agricultural students by means of the "Foreign Language" course ]. In : Vestnik FGOU VPO "Moskovskiy gosudarstvennyy agroinzhenernyy universitet imeni V.P. Goryachkina" : nauch. zhurnal. - Issue 5 (36). Teoriya i metodika professional' nogo obrazovaniya / Ed. by P. F. Kubrushko. - M. : FGOU VPO MGAU. pp. 83-87.

VERBITSKIY, A.A. 2007.4 Inoyazychnyye kompetentsii kak komponent obshchey professional' noy kompetentsii inzhenera: problemy formirovaniya [Foreign competences as a component of an engineer' s overall professional competence: problems of development]. Vyssheye obrazovaniye segodnya. n. 12, pp. 27-31.

YEREGINA, S.V. 2006. Professional' naya oriyentatsiya studentov fizkul' turnykh vuzov [Career guidance of physical education students]: DSc (Ed) thesis. - M. 443 p. ZIMNYAYA, I.A. 2010. Formirovaniye i otsenka sformirovannosti sotsial' nykh kompetentnostey u studentov vuzov pri osvoyenii novogo pokoleniya OOP VPO [Development and assessment of social competencies in university students for the elaboration of a new generation of higher education curricula]. - M.: Issledovatel' skiy tsentr problem kachestva podgotovki spetsialistov. $40 \mathrm{p}$.

Words: 7578

Characters: 54745 (30,41 standard pages)

Assoc. Prof. Aleksei Yu. Alipichev, PhD

Foreign Languages Department

Russian State Agrarian University - Moscow Timiryazev Agricultural Academy

Timiryazevskaya Str., 49

127550 Moscow

Russia

al_new2003@mail.ru

Assoc. Prof. Irina P. Gotovtseva, PhD

Foreign Languages Department

Russian State Agrarian University - Moscow Timiryazev Agricultural Academy 
Timiryazevskaya Str., 49

127550 Moscow

Russia

rgauinyaz@ rgau-msha.ru

Assoc. Prof. Olga V. Takanova, PhD

Foreign Languages Department

Russian State Agrarian University - Moscow Timiryazev Agricultural Academy Timiryazevskaya Str., 49

127550 Moscow

Russia

olgatakanova@yandex.ru 